\title{
Spectroscopic Detection of an Extraordinary Flaring-Event on DF Tau
}

\author{
J. Z. Li ${ }^{1,2}$, W. P. Chen ${ }^{1}$ \& W. H. Ip ${ }^{1}$
}

1. Institute of Astronomy, National Central University, Chung-Li 32054 (Email: ljz@astro.ncu.edu.tw)

2. Beijing Astronomical Observatory, National Astronomical Observatory, Chinese Academy of Sciences, Beijing 100012, China

\begin{abstract}
An accretion intrigued flare-like brightening of DF Tau has been recorded spectroscopically, during which spectacular spectral changes took place. We inferred the possible formation of a transient envelope during the process of this episodic mass accretion of DF Tau.
\end{abstract}

\section{Motivation of Study}

With an initial motivation to study properties of hot spots on Classical T Tauri stars (CTTS), we have launched a spectroscopic monitoring campaign to study some CTTS known to display periodic or quasi-periodic light variations. DF Tau is one of the program stars.

\section{Observations and Data Reduction}

Intermediate resolution spectroscopic observations of DF Tau and several other CTTS (with a dispersion of $50 \mathrm{~A} / \mathrm{mm}, 1.2 \mathrm{~A} /$ pixel and a 2.5 "slit) were carried out by the $2.16 \mathrm{~m}$ optical telescope of the Beijing Astronomical Observatory, in both the blue (3500A - 4700A) and red (5750A - 6950A) spectral ranges on a nightly basis. Each night, when all the program stars were exposed (once per CTTS) with the blue settings, the red settings were employed, or vise versa. An OMR (Optomechanics Research Inc.) spectrograph and a Tektronix $1 \mathrm{Kx} 1 \mathrm{~K}$ CCD detector were used during the observations from Jan. 7 to Jan. 14, 2000 (Jan. 11 was skipped for weather reasons).

The spectral data were reduced following standard procedures in the NOAO Image Reduction and Analysis Facility (IRAF, version 2.11) software packages.

\section{Results and Discussion}

1) Our 8-night campaign of these spectroscopic observations of DF Tau witnessed the entire event of an extraordinary flare-like brightening, with a remarkably sharp increase in brightness (about 4 magnitudes within one day in the B band), followed by a slow decay lasting for 5 days. 


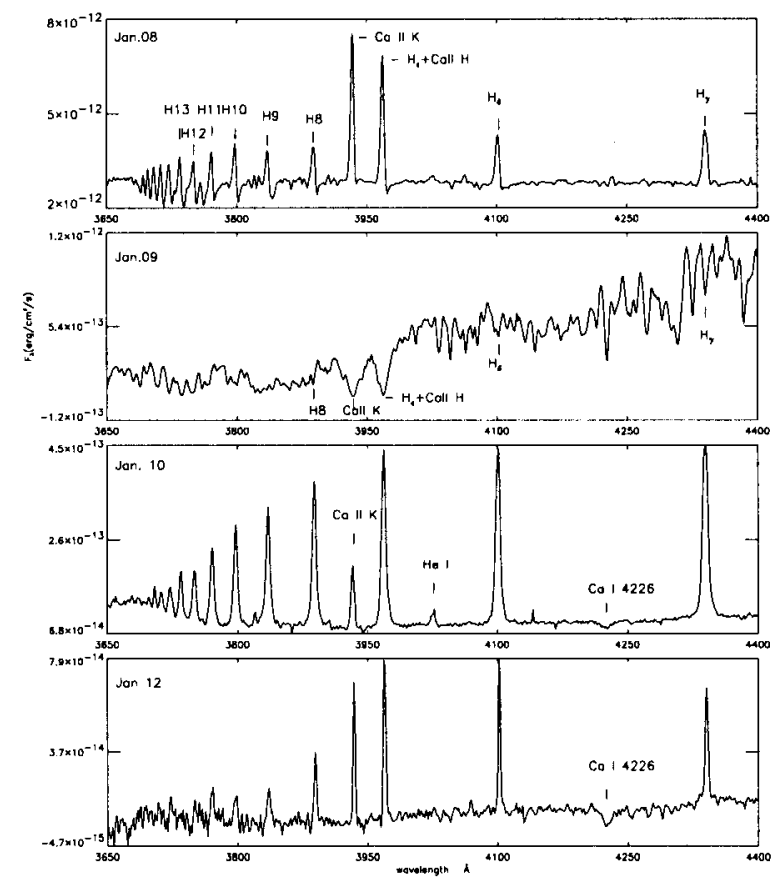

Figure 1. Representative spectra of this brightening of DF Tau.

2) Spectacular changes took place between Jan. 8 and 9 in the continuum and line emissions, a major difference is that the latter seems to have lost its UV excess, while apparent excessive emissions still exist, which could be due to wavelength-dependent cooling efficiency as well as probable severe absorption encountered along the line of sight (Fig. 1). This could indicate the formation of a transient envelope, which serves just like an extra extension of the photosphere, where severe absorption takes place.

3) The amplitude of variation during this event reached up to 6 magnitudes in the $\mathrm{B}$ band and decreased as a function of wavelength to about 2 magnitudes in the $\mathrm{V}$ and $\mathrm{R}$ bands.

4) Apparent correlation between variations of line flux and veiling is displayed in this run of observations of DF Tau.

\section{Summary}

1) An unprecedentedly large flare-like event was recorded spectroscopically on DF Tau. 2) Significant Y Y Orionis line profiles (Inverse P Cygni profiles), of supersonic origin, appeared on Jan. 8, suggesting inhomogeneous mass infall onto the contracting star. 3) We suggest the probable formation of a transient envelope in or over the shock-cooling region, which caused the spectacular spectral changes between Jan. 8 and 9, and its rapid, radial dissipation, which may have coherent relations to its intrinsic fierce turbulence (as indicated by the unusually extended CaII H \& K absorption on Jan. 9 and broad Balmer emission lines on Jan. 10) and also the later emerging stellar wind. 